\title{
A near Fourier-transform limited optical parametric oscillator/amplifier system as a pulsed source for high resolution spectroscopy
}

\author{
K.G.H. Baldwin ${ }^{1}$, M. Kono ${ }^{1}$, Y. He ${ }^{2}$, R. T. White ${ }^{2}$, B. J. Orr ${ }^{2}$, \\ ${ }^{1}$ Research School of Physical Sciences and Engineering, The Australian National University, \\ Australia; ${ }^{2}$ Centre for Lasers and Applications, Macquarie University, Sydney, Australia \\ Kenneth.Baldwin@anu.edu.au
}

\begin{abstract}
We use optical heterodyne and nonlinearoptical spectroscopic techniques to measure the near Fourier-transform limited output from a narrowband tunable injection-seeded, pulsed optical parametric oscillator/amplifier system that has a controllable frequency chirp of $<10 \mathrm{MHz}$.
\end{abstract}

We have demonstrated single-longitudinal-mode (SLM) operation of an injection-seeded, tunable optical parametric oscillator (OPO) based on quasi-phasematched $\mathrm{KTiOPO}_{4}$ (PPKTP) [1]. Our most recent system [2] employs a SLM Nd:YAG laser to pump the PPKTP crystal with much longer ( 27-ns) 532-nm pulses, and with injection-seeding at $\sim 842 \mathrm{~nm}$ by a cw SLM tunable diode laser (TDL). This generates narrowband, continuously tunable output radiation with 25-ns pulse duration. The optical layout of our system is shown in Fig. 1.

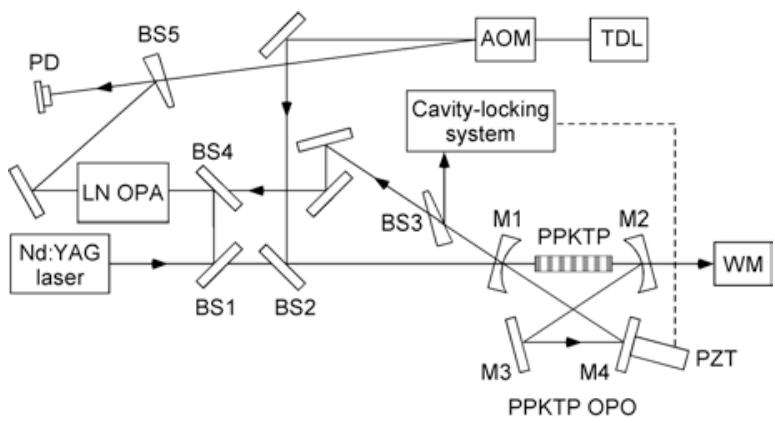

Fig. 1. Schematic of the long-pulse optical parametric oscillator/amplifier (OPO/OPA) and its optical heterodyne detection system. AOM: acousto-optic modulator $(\sim 730 \mathrm{MHz})$. BS1-5: beam splitters. LN: $\mathrm{LiNbO}_{3}$; M1-4: OPO cavity mirrors. PD1: fast photodetector. PZT: piezoelectric translator. WM: pulsed wavemeter.
Optical heterodyne techniques [3] have been used to control the OPO's output frequency chirp and to optimize its SLM operation. Investigation of the longpulsed system [2] shows that at low pump energies $(\leq 2.5$ times the free-running threshold), the narrowband tunable OPO output is SLM and its frequency chirp can be $<10 \mathrm{MHz}$, much less than the transform-limited optical bandwidth $(\sim 17.5 \mathrm{MHz})$. Fig. 2 shows that the chirp (variation of the instantaneous frequency $f_{\text {inst }}$ during each OPO pulse) can be minimized by matching the output wavelength of the injection-seeded OPO to that of a free-running (unseeded) OPO with a given PPKTP crystal temperature.

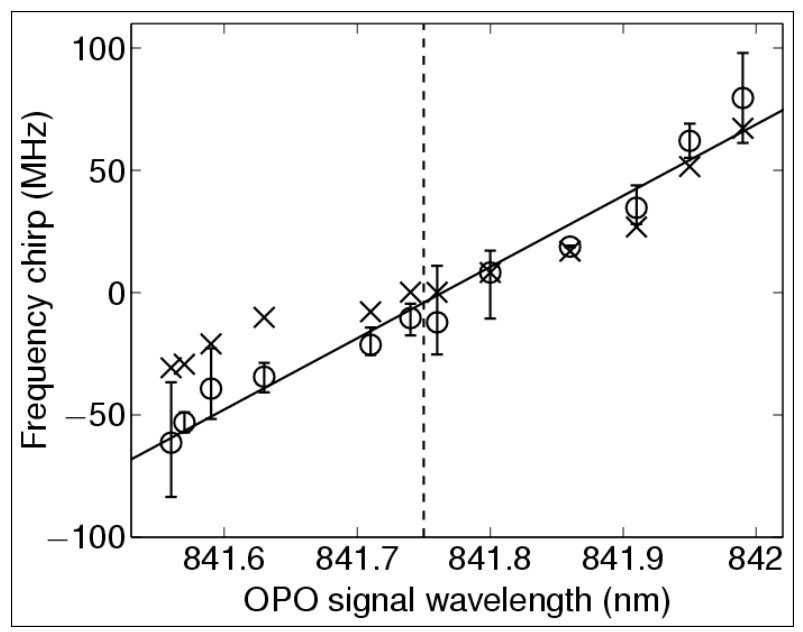

Fig. 2. Frequency chirp as a function of OPO signal wavelength $1_{\mathrm{s}}$ using our alternative linear-fit $(x)$ and $\Delta f_{\text {inst }}($ o) definitions $[2,3]$. The solid line is an unweighted straight-line fit to the $\Delta f_{\text {inst }}$ data, with error bars to show the scatter of $\Delta f_{\text {inst }}$ values in each data set. The fit has a slope of $292 \pm 17 \mathrm{MHz} \mathrm{nm}{ }^{-1}$ and it predicts a minimum-chirp wavelength of $841.76 \mathrm{~nm}$. The vertical dashed line indicates the freerunning OPO signal wavelength, $\lambda_{\text {free }}(841.75 \mathrm{~nm})$. 
More recently, we have amplified the OPO output using an optical parametric amplifier (OPA) based on birefringently phase-matched $\mathrm{LiNbO}_{3}$. Chirp characterization experiments indicate that the amplified output does not appreciably degrade the OPO output frequency performance.

Furthermore we have used the output from this advanced PPKTP OPO/OPA system injection-seeded at $\sim 822 \mathrm{~nm}$ by a Ti:sapphire laser to measure the fluorescence from Doppler-free two-photon excitation of cesium vapor. In Fig. 3 we compare the width of the two-photon excitation spectrum $2 \Delta v_{\mathrm{b}}$ (open symbols) with the Fourier transform (FT) of the pulse power temporal profile $\Delta t_{2}$ (solid symbols).

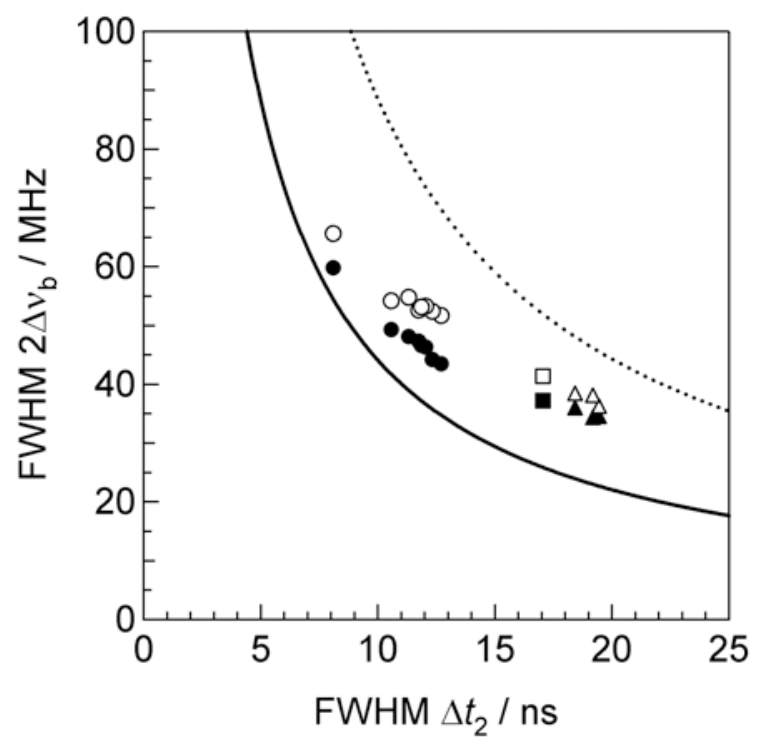

Fig. 3. Plots as a function of the FWHM $\Delta t_{2}$ of the square of the temporal power profile: open symbols - the FWHM $2 \Delta v_{\mathrm{b}}$ of the Cs TPE linewidths; closed symbols - FWHM of the FT-limited TPE power spectra as calculated, e.g., in Fig. 2(b). Triangles - OPO only (varying chirp); square - OPA/2 (zero chirp); circles - full OPA (varying chirp). Also shown are the FT limits for Gaussian (solid line) and rectangular (broken line) temporal power profiles.
The values are plotted as a function of the FWHM of the square of the pulse power temporal profile (for this twophoton process), which decreases with increasing chirp and amplification. We employed full, half and no amplification of the OPO as indicated. The FT limited values for idealized Gaussian and rectangular profiles with equivalent FWHM are also shown for comparison.

The results indicate that the measured bandwidth of the Cs two-photon excitation is very close to the calculated FT limited values for each pulse profile. The narrowest linewidths occur for the zero-chirp measurements - the right-most points in each amplifier data set. In the case of the zero chirp (right most) OPO-only data point, the signal output was within a few per cent of the FT limit.

There is therefore a direct correlation between increasing chirp and decreasing pulse duration, accompanied by increasing linewidth. However, the chirp can easily be controlled to be very close to zero simply by matching the seed laser wavelength to the free-running OPO wavelength [1-4]. Therefore, this system has great potential for applications that require very high resolution, near FT-limited pulsed laser spectroscopy.

\section{REFERENCES}

[1] R. T. White, Y. He, B. J. Orr, M. Kono, and K. G. H. Baldwin, "Pulsed injection-seeded optical parametric oscillator with low frequency chirp for high-resolution spectroscopy," Opt. Lett. 28, 12481250 (2003).

[2] R. T. White, Y. He, B. J. Orr, M. Kono, and K. G. H. Baldwin, "Control of frequency chirp in nanosecond-pulsed laser spectroscopy. 2. A long-pulse optical parametric oscillator for narrow optical bandwidth," J. Opt. Soc. Am. B, 21, 1586-1594 (2004).

[3] R. T. White, Y. He, B. J. Orr, M. Kono, and K. G. H. Baldwin, "Control of frequency chirp in nanosecond-pulsed laser spectroscopy. 1. Optical-heterodyne chirp analysis techniques," J. Opt. Soc. Am. B, 21, 1577-1585 (2004).

[4] R. T. White, Y. He, B. J. Orr, M. Kono, and K. G. H. Baldwin, "Transition from single-mode to multimode operation of an injectionseeded pulsed optical parametric oscillator," Optics Express 12, 5655 5660 (2004). 